\title{
Copper oxide nanoparticles-loaded zeolite and its characteristics and antibacterial activities
}

\begin{abstract}
In the present work, a simple and green co-precipitation method was used to prepare copper oxide-zeolite nanocomposites (CuO-zeolite NCs). The weight ratio (1, 3, 5, 8 and $10 \mathrm{wt} \%)$ of $\mathrm{CuO}$ nanoparticles (NPs) loaded into zeolite was investigated to obtain the optimum $\mathrm{CuO}$ distribution for antibacterial activities. The prepared $\mathrm{CuO}$-zeolite NCs were characterized by ultraviolet-visible (UV-vis) spectroscopy, Fourier transform infrared (FT-IR) spectroscopy, powder X-ray diffraction (XRD), and energy dispersive X-ray fluorescence spectrometry (EDXRF). The transmission electron microscopy (TEM) and field emission scanning electron microscopy (FE-SEM) revealed a uniform surface morphology of the CuO-zeolite NCs. The UV-vis spectrum of NCs showed absorption peaks between 230 and $280 \mathrm{~nm}$ for nano-CuO in the XRD patterns, and new peaks appeared between $\left(36.56^{\circ}-38.83^{\circ}\right)$ related to the $\mathrm{CuO}$. At weight ratio less than $10 \mathrm{wt} \%$, the $\mathrm{CuO}$ nanoparticles loaded to the zeolite exhibited spherical shapes with average particle diameter of $6.5 \mathrm{~nm}$ measured by TEM and XRD. Antibacterial activities were tested against Gram-negative and Gram-positive bacteria. The obtained results showed that, $\mathrm{CuO}$-zeolite $\mathrm{NCs}$ with $8 \mathrm{wt} \% \mathrm{CuO}$ nanoparticles had the highest antibacterial activities against Bacillus Subtilis B29 and Salmonella Choleraesuis ATCC 10708, which can be attributed to the good dispersion of $\mathrm{CuO}$ NPs on the zeolite surface.
\end{abstract}

Keyword: Copper oxide-zeolite; Nanocomposites; Antibacterial 